\title{
Influencia del apoyo por parte del terapeuta en la satisfacción de un programa auto-aplicado a través de Internet para la depresión
}

\author{
Clara Miguel SANZ \\ al189588@uji.es \\ LAURA DÍAZ SANAHUJA \\ al189588@uji.es \\ AdRIANA MIRA PASTOR \\ al189588@uji.es \\ JUANA MARÍA BRETÓN LÓPEZ \\ al189588@uji.es \\ CRistina Botella ARBona \\ al189588@uji.es
}

\section{Resumen}

Introducción: Las intervenciones auto-aplicadas a través de Internet cuentan con resultados consistentes respecto a su eficacia como tratamiento psicológico. Sin embargo, las altas tasas de abandono siguen suponiendo un reto. El apoyo recibido por parte del terapeuta a lo largo de la intervención es una de las variables que pueden influir en estas tasas (Karyotaki et al., 2017). Objetivo: Este estudio pretende analizar las diferencias en satisfacción, agrado y utilidad respecto a recibir o no apoyo telefónico en una intervención auto-aplicada a través de Internet para la depresión. Método: 80 participantes con síntomas depresivos fueron aleatorizados en dos condiciones experimentales: Intervención auto-aplicada con apoyo $(\mathrm{N}=44)$ y sin apoyo telefónico $(\mathrm{N}=$ 36). En el post-tratamiento se evaluó el grado de satisfacción con la intervención, así como el agrado y utilidad de las llamadas. Resultados: Ambos grupos valoraron, sin diferencias significativas entre ellos, que estaban satisfechos con el tratamiento. Únicamente se observaron diferencias significativas $(F=4,67 ; p=0,035)$ en relación a la aversividad del mismo, valorándolo como más aversivo el grupo sin apoyo telefónico. El grupo que contaba con el contacto telefónico lo evaluó con un alto porcentaje de agrado y utilidad, mientras que el grupo sin apoyo no valoró tan positivamente la posibilidad de haber dispuesto de éste. Conclusiones: Los resultados obtenidos sugieren la importancia de ofrecer apoyo telefónico durante la intervención auto-aplicada, sin embargo, también plantean que, en los programas con los que el usuario se siente satisfecho, el apoyo explícito se vuelve menos necesario.

Palabras clave: intervención auto-aplicada, Internet, depresión, apoyo telefónico. 


\section{Abstract}

Introduction: Self-applied interventions via the Internet have shown consistent results regarding their efficacy as psychological treatment. However, they are characterized by a high dropout rate. This issue can be influenced by the support the therapist offers throughout the intervention (Karyotaki et al., 2017). Objective: This study intends to analyse whether "satisfaction», liking and «utility» indexes are different between patients receiving or not this telephone support during a self-applied intervention for depression via the Internet. Methods: 80 participants presenting depressive symptoms were randomized in two experimental conditions: Self-applied interventions via the Internet with and without telephone support ( $\mathrm{N}=44$ and $\mathrm{N}=36$, respectively). When the treatment was finished, satisfaction with the intervention, and liking and utility degree of these calls were evaluated. Results: No significant differences were observed between groups in terms of satisfaction, being both groups satisfied with the treatment. Significant differences were only found on treatment aversion $(F=4.67 ; p=0.035)$, being considered more aversive by the group without telephone support. The group that received telephone support assessed this human intervention with a high percentage of satisfaction and utility, while the group without this kind of support did not appreciate the possibility of having this contact. Conclusions: Present results suggest the importance of offering support during the self-applied intervention. However, they also point out that in programs in which participants feel satisfied, explicit support becomes less necessary.

Keywords: self-applied intervention, Internet, depression, human support.

\section{Introducción}

La depresión constituye uno de los problemas más importantes para la salud mundial e interfiere de manera significativa en distintas áreas de funcionamiento del individuo (Haro et al., 2013). El número total de personas con depresión a nivel mundial incrementó en un 18,4\% entre 2005 y 2015, estimándose una prevalencia total del 4.4\% (OMS, 2017). Asimismo, supone un coste económico muy elevado a nivel de salud pública (Buntrock et al., 2016) y tiene un impacto muy significativo en el índice de calidad de vida ajustada por años de vida (YLD), siendo éste de 8,1\% en Europa y de 8,7\% en España (OMS, 2017).

Actualmente, contamos con tratamientos psicológicos eficaces y empíricamente validados para la depresión como la terapia cognitivo-conductual, la terapia conductual y la terapia interpersonal (Nathan \& Gorman, 2016). Sin embargo, estas intervenciones tienen una importante limitación: los servicios de salud mental con los que contamos no son adecuados en términos de accesibilidad y calidad. Menos del 50 \% de las personas con depresión son tratadas por un profesional de la salud y sólo un cuarto recibe el tratamiento adecuado, bien sea por los costes y las demandas de la terapia cara a cara, el tiempo requerido en su aplicación o la falta de profesionales bien entrenados (Titov, 2011). A esto se suma que, aquellos que reciben el tratamiento, por lo general, lo hacen después de mucho tiempo, una vez el problema ha generado efectos adversos en sus vidas, por lo que el éxito de la intervención es mucho más difícil que si la ayuda se hubiera ofertado en el momento oportuno (Kessler \& Greenberg, 2002). 
Los datos anteriores ponen de manifiesto que el modelo dominante de terapia con el que contamos, la terapia cara a cara, no soluciona estas necesidades (Kazdin \& Blase, 2011). Con el fin de potenciar la accesibilidad a aquellas personas que lo necesitan y que debido a la distancia o coste no recibirían tratamiento de otro modo, se plantea como estrategia fundamental la inclusión de las Tecnologías de la Información y la Comunicación (TICs) (Kazdin \& Rabbitt, 2013). Especialmente el uso de Internet en la aplicación de los tratamientos psicológicos ha mostrado ser una estrategia efectiva (Cuijpers, Donker, van Straten \& Andersson, 2010; Karyotaki et al., 2017). Los estudios de meta-análisis ponen de manifiesto que estos tratamientos tienen tamaños del efecto comparables a los de los tratamientos cara a cara (Bee et al., 2008; Cuijpers et al., 2010), mostrando la utilidad de numerosas intervenciones autoaplicadas a través de Internet para la depresión sin que pierdan su eficacia (Karyotaki et al., 2017) y siendo intervenciones costo-efectivas (Donker et al., 2015).

Asimismo, la actitud de los pacientes hacia este tipo de tratamientos es similar a la que muestran ante las terapias cara a cara (Moritz, Schröder, Meyer, \& Hauschildt, 2013). A pesar de todas estas ventajas, todavía se encuentran importantes problemas que afrontar ante este tipo de tratamientos, como es la elevada tasa de abandonos. Estudios de meta-análisis sobre programas auto-aplicados a través de Internet para la depresión muestran una tasa de abandonos de alrededor del 32\% (Melville, Casey, \& Kavanagh, 2010). Esto puede deberse a múltiples causas, siendo posiblemente una de ellas el apoyo proporcionado por parte del terapeuta a lo largo de la intervención auto-aplicada). Recientemente un número importante de investigaciones se han llevado a cabo para determinar el papel del apoyo humano en estas intervenciones auto-aplicadas.Los estudios de meta-análisis consideran que los tratamientos más eficaces y con menos abandonos son los que cursan con apoyo por parte del terapeuta, en comparación con los programas totalmente auto-aplicados (Karyotaki et al., 2017). No obstante, existe evidencia de que las intervenciones totalmente auto-aplicadas también son eficaces en el tratamiento de la depresión y son mucho más fáciles de implementar y menos costosas que las intervenciones auto-aplicadas con apoyo (Karyotaki et al., 2015). Por todo ello es interesante conocer la opinión de las personas que utilizan los programas auto-aplicados en cuanto a recibir o no dicho apoyo a lo largo de la intervención.

Con el objetivo de conocer más específicamente el papel que tiene el apoyo del terapeuta en los tratamientos auto-aplicados, en este estudio se plantean las siguientes cuestiones: ¿Es necesario el apoyo semanal por parte de un terapeuta durante la realización de un programa de intervención auto-aplicado a través de Internet para que los usuarios estén satisfechos y valoren positivamente la intervención? ¿Cómo valoran el apoyo y qué utilidad tiene para ellos?

El objetivo del presente estudio es analizar las diferencias en satisfacción, agrado y utilidad respecto a recibir o no apoyo telefónico por parte del terapeuta en una intervención autoaplicada a través de Internet para la depresión.

\section{Método}

\section{Participantes}

La muestra está compuesta por 80 participantes con síntomas de depresión leve a moderada, con puntuación igual o inferior a 28 en el Inventario de Depresión de Beck (Beck, Steer \& Brown, 1996). La media de edad de la muestra fue de 35,15 años, con una desviación típica de 9,45 . Para poder participar en el estudio se debía tener entre 18 y 65 años, posibilidad de utilizar ordenador con Internet en casa y presentar síntomas de depresión leve o moderada. 
Estar recibiendo tratamiento psicológico o haber recibido en el último año fueron criterios de exclusión, además de padecer un trastorno mental grave en el Eje I o la presencia de planes o ideación suicida.

\section{Tratamiento}

Los participantes recibieron un programa totalmente auto-aplicado a través de Internet: Sonreír es Divertido (www.sonreiresdivertido.com). Este programa tiene dos objetivos fundamentales, la prevención y el tratamiento de la depresión, ya que ofrece asistencia tanto a personas con alto riesgo de padecer depresión como a personas que ya presentan la sintomatología. El programa de intervención es de corte cognitivo-conductual. Incorpora estrategias para mejorar el estado de ánimo positivo, así como los enfoques más tradicionales para prevenir el estrés y la depresión, como la activación conductual. Combina los procedimientos existentes más efectivos para promocionar la regulación emocional, la capacidad de afrontamiento y la resiliencia.

Sonreír es Divertido está diseñado para que se lo pueda aplicar uno mismo, desde casa o desde cualquier lugar que disponga de acceso a Internet. El programa está formado por los siguientes componentes terapéuticos: motivación para el cambio, psicoeducación, terapia cognitiva, activación comportamental, psicología positiva y prevención de recaídas. Estos componentes son trabajados por el paciente a lo largo de los 8 módulos que conforman el protocolo de tratamiento. Para más detalle, ver Figura 1. Además de los componentes de la TCC, se incluyen ejercicios prácticos y estrategias para mejorar el afecto positivo. El propio programa también ofrece apoyo e información a los usuarios para que realicen un seguimiento de su estado dentro del tratamiento. Para ello utilizan tres herramientas transversales: ¿Cómo estoy?, donde se ofrecen gráficas, para ver la evolución de sus puntuaciones después de haber cumplimentado cada módulo (afecto positivo y negativo, ansiedad y depresión); el Calendario, donde se muestra la frecuencia de acceso al sistema y las tareas realizadas o pendientes; y el Diario de actividad, que muestra la relación entre el estado de ánimo y las actividades realizadas durante la semana.
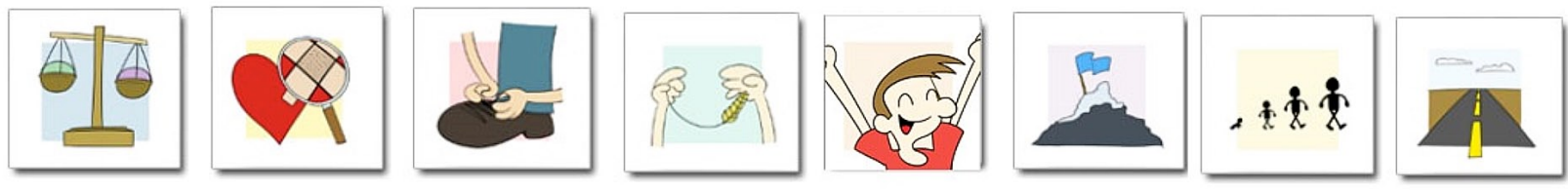

1. Motivación para el cambio

2. Entendiendo los problemas emocionales

3. Aprendiendo a ponerse en marcha

4. Aprendiendo a ser flexibles

5. Aprendiendo a disfrutar: importancia de las experiencias positivas

6. Aprendiendo a vivir: importancia de los valores y las metas

7. Viviendo $y$ aprendiendo

8. ¿Y a partir de ahora...qué?

Figura 1. Módulos del programa Sonreír es Divertido 


\section{Diseño}

Se utilizó un estudio controlado aleatorizado de simple ciego en el que los 80 participantes fueron asignados al azar en dos condiciones experimentales:

1) Intervención auto-aplicada con apoyo telefónico (SCA, $N=44)$. En esta condición los participantes realizaban el programa de tratamiento Sonreír es Divertido de forma totalmente auto-aplicada a través de Internet. A lo largo de la intervención, los participantes de esta condición recibían una llamada semanal por parte de un terapeuta de una duración máxima de dos minutos. El objetivo de estas llamadas era valorar si el uso del programa se realizaba correctamente, recomendarles avanzar a un ritmo adecuado y fomentar la importancia de practicar las tareas que se presentan en los diferentes módulos (Figura 2).

El contenido de la llamada cambiaba en función del progreso:

1. Si el participante no ha finalizado el módulo (Motivarlo para hacerlo)

2. Si el participante ha finalizado uno de los módulos (Refuerzo).

3. Si el participante ha finalizado dos módulos (Refuerzo y recomendación de ralentizar la ejecución).

4. Si el participante finaliza más de dos módulos (Recomendación de ralentizar la ejecución)

Recordarle realizar las tareas que se piden en cada uno de los módulos

Figura 2. Protocolo de apoyo telefónico

2) Intervención auto-aplicada sin apoyo telefónico (SSA, N = 36). En esta condición, los participantes recibían el mismo programa de intervención, Sonreír es Divertido, aunque sin la llamada de apoyo semanal por parte del terapeuta.

Ambas condiciones de intervención cuentan con las tres herramientas transversales descritas anteriormente.

\section{Medidas}

Escala de Satisfacción sobre el Tratamiento (Adaptación Borkovec \& Nau, 1972): Los aspectos que se abordan en esta escala son: 1) La lógica del tratamiento; 2) La satisfacción con el mismo; 3) El grado en que lo recomendarían a un amigo con el mismo problema; 4) El grado en el que consideran que le ha ayudado en otros problemas; 5) El grado en que les ha sido útil en su caso; 6) El grado en que consideran que el tratamiento ha sido aversivo.

Entrevista cualitativa (Utilizada en el estudio de Mira, 2014): Se elaboró una entrevista cualitativa para valorar la opinión de los participantes acerca de la utilidad y agrado de las llamadas de apoyo semanal. Estas preguntas se contestaban en una escala tipo likert de 1 «poquísimo» a 5 «muchísimo». 


\section{Procedimiento}

En primer lugar, se realizó un muestreo telefónico para comprobar que los participantes cumplieran los criterios de inclusión del estudio. Posteriormente, en una entrevista presencial, se evaluaron los criterios de inclusión, se administró el BDI-II para evaluar el nivel de depresión y se realizó una entrevista diagnóstica para descartar la presencia de otros trastornos psicológicos graves. Una vez pasaron a formar parte del estudio, los participantes fueron asignados aleatoriamente a las dos condiciones experimentales. Después, un terapeuta contactó con ellos para informarles acerca de la condición a la que habían sido asignados y de las fases posteriores. Una vez tenían una cuenta y una contraseña, podían acceder a la plataforma y realizar la evaluación pre-tratamiento. Posteriormente realizaban cada uno de los módulos de intervención. Se realizaba una evaluación continua tras finalizar cada módulo en la que se evaluaba sintomatología ansiosa, depresiva y se rastreaba la presencia de ideación suicida. Después del módulo 2, los participantes cumplimentaban la Escala de Expectativas sobre el tratamiento.

Al finalizar los 8 módulos del programa los participantes realizaron la evaluación posttratamiento y se evaluó en ambos grupos el grado de satisfacción con la intervención a través de la Escala de Satisfacción sobre el Tratamiento. Adicionalmente, mediante la entrevista cualitativa, al grupo SCA se le preguntó en qué medida había sido de agrado la llamada de apoyo telefónico por parte del terapeuta y cómo de útil la consideraban. Al grupo SSA se le preguntó en qué medida le hubiera resultado de agrado una llamada de apoyo telefónico por parte del terapeuta y cómo de útil la hubieran considerado.

\section{Resultados}

\section{Satisfacción}

Para estos análisis se cuenta con las puntuaciones en satisfacción de 28 de los participantes de la condición sin apoyo y de 36 de los participantes de la condición con apoyo. Son los datos de los participantes que realizaron la evaluación post-tratamiento en la que se administraba la Escala de Satisfacción sobre el Tratamiento (Adaptación Borkovec \& Nau, 1972). En general, los resultados obtenidos en satisfacción con el tratamiento fueron muy elevados en todas las variables evaluadas en la escala, con un valor de media en el post-tratamiento entre 7 y 8 puntos sobre 10. Los análisis ANOVA de un factor (ver Tabla 1) indicaron que ambos grupos valoraron, sin diferencias significativas entre ellos, que estaban satisfechos con el tratamiento, que fue lógico, útil y que lo recomendarían a un amigo. Únicamente se encontraron diferencias significativas $(F=4,67 ; p=0,035)$ en relación a la aversividad del tratamiento, valorándolo como más aversivo el grupo sin apoyo. No obstante, las puntuaciones medias en dicho ítem (SCA = 1,67 ; SSA $=2,75)$ indican que los participantes valoraron el tratamiento como poco aversivo en ambas condiciones. 
Tabla 1

Medidas de la satisfacción con el tratamiento de las dos condiciones de intervención analizadas utilizando ANOVAs de un factor

\begin{tabular}{lccccc}
\hline VD & Condición & $\mathrm{M}$ & $\mathrm{DT}$ & $\mathrm{F}$ & $p$ \\
\hline Lógico & SSA & 8,21 & 1,77 & 0,109 & 0,742 \\
& SCA & 8,08 & 1,40 & & \\
Sotal & 8,14 & 1,56 & & 0,830 \\
& SSA & 7,93 & 1,92 & 0,046 & \\
Recomendacción & SCA & 7,83 & 1,61 & & 0,761 \\
& Total & 7,88 & 1,74 & & \\
Útil otros problemas & SSA & 8,25 & 1,96 & 0,093 & 0,818 \\
& SCA & 8,39 & 1,68 & & \\
Útil en su caso & Total & 8,33 & 1,79 & & 0,713 \\
& SSA & 8,04 & 1,92 & 0,053 & \\
Aversivo & SCA & 8,14 & 1,66 & & \\
& Total & 8,09 & 1,76 & & 0,035 \\
& SSA & 7,46 & 2,03 & 0,137 & \\
\hline
\end{tabular}

Nota. VD = Variable dependiente; $M=$ Media; $D T=$ Desviación típica; $F=$ valores de $F ; p=$ grado de significación; $S S A=$ Condición experimental sin apoyo por parte del terapeuta $(n=28)$; SCA = Condición experimental con apoyo por parte del terapeuta $(n=36)$.

\section{Utilidad}

A los participantes de la condición con apoyo por parte del terapeuta se les preguntó en qué medida habían considerado útil la llamada de apoyo semanal. La mayoría de los participantes de esta condición (SCA, N = 48) consideraron que la llamada les había sido útil («muchísimo» $=50 \%$ y «mucho» $=27,08 \%$ ). Al mismo porcentaje de los participantes les resultó «algo» útil y «muy poco útil» la llamada (8,33 \%) y el 6,23 \% la valoraron con «poquísima» utilidad (ver Figura 3).

En cuanto a los participantes de la condición experimental sin apoyo por parte del terapeuta, se les preguntó en qué medida habrían considerado útil dicho apoyo. La mayoría de estos participantes (SSA, $\mathrm{N}=34$ ) consideraron que les habría resultado útil («muchísimo» = $23,52 \%$ y «mucho» $=23,52 \%)$. Un 17,65 \% consideraron que les habría resultado "algo» útil y un $26,47 \%$ consideró que les habría sido «poco» útil. Sólo tres un $8,82 \%$ la valoraron con «poquísima» utilidad (ver Figura 4).

\section{Agrado}

A los participantes de la condición experimental con apoyo (SCA, $N=48$ ) se les preguntó en qué medida les había gustado recibir la breve llamada de apoyo semanal. La mayoría 
contestó que les gustó («muchísimo» $=47,92 \%$, «mucho» $=29,17 \%$ ), el 18,75 \% valoraron que les gustó «algo» recibir la breve llamada de apoyo, sólo a dos participantes les gusto «poco» $(2,08 \%)$ y a otros dos «poquísimo» (2,08 \%) (Figura 5$)$.

A los participantes de la condición experimental sin apoyo (SSA, $N=34$ ) se les preguntó en qué medida les habría gustado recibir la llamada telefónica. El 29,41 \% de los participantes de esta condición indicó que les habría gustado "muchísimo» recibirla y un $14,71 \%$ que les habría gustado «mucho». A un 20,59 \% le habría gustado «algo» y a un 29,41 \% les habría gustado «poco» recibirla. Sólo a dos participantes $(5,88 \%)$ les habría gustado «poquísimo» que les hicieran una breve llamada semanal (ver Figura 6).

Utilidad SCA

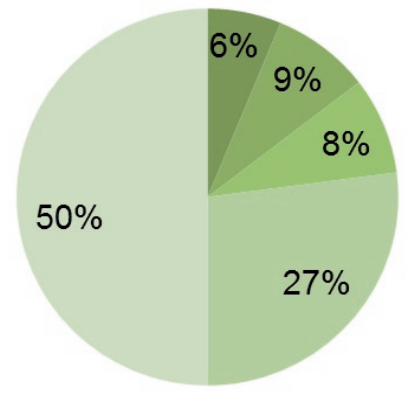

Figura 3. Porcentajes de valoración de la utilidad de la llamada de apoyo de los participantes de la condición SCA
Utilidad SSA

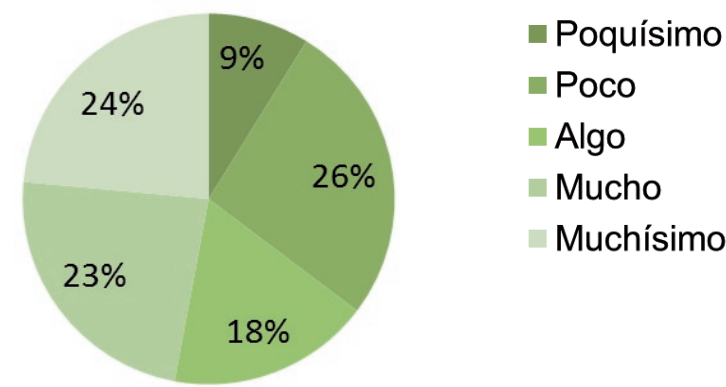

Figura 4. Porcentajes de valoración de la utilidad de la llamada de apoyo de los participantes de la condición SSA.

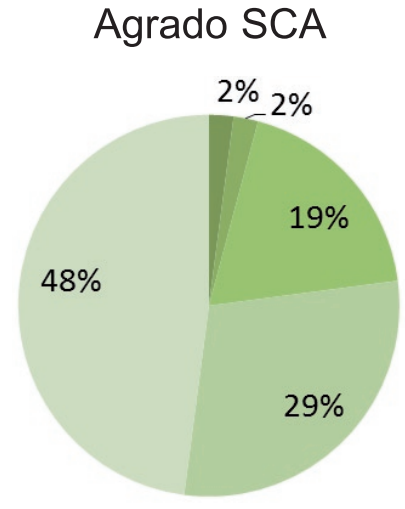

Figura 5. Porcentajes de agrado de las llamadas de apoyo de los participantes de la condición SCA.
Agrado SSA

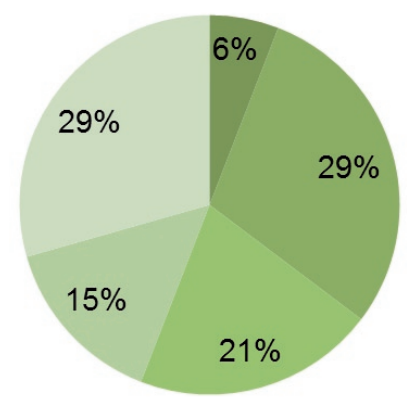

Figura 6. Porcentajes de agrado de las llamadas de apoyo de los participantes de la condición SSA.

\section{Discusión y conclusiones}

El objetivo de este estudio era analizar las diferencias en satisfacción, agrado y utilidad respecto a recibir o no apoyo telefónico por parte del terapeuta en una intervención autoaplicada a través de Internet para la depresión. 
Los resultados obtenidos en cuanto a satisfacción muestran que los participantes de ambas condiciones experimentales se sintieron muy satisfechos con el tratamiento, no evidenciándose diferencias significativas entre ambos grupos a excepción de la aversividad del tratamiento. En este ítem, el grupo sin apoyo lo consideró más aversivo, aunque las puntuaciones en esta variable fueron muy bajas para ambos grupos. Esta falta de diferencias significativas en satisfacción entre una condición que recibe apoyo humano y otra que sólo cuenta con el apoyo automatizado que ofrece el programa se ha obtenido también en un estudio reciente (Kelders, Bohlmeijer, Pots \& van Gemert-Pijnen, 2015). Una posible explicación de la falta de diferencias entre los grupos puede ser la riqueza y calidad del apoyo ofrecido por Sonreír es Divertido a través de sus herramientas transversales de apoyo (¿Cómo estoy?, Calendario y Diario de Actividad). El programa ofrece una retroalimentación personalizada y continua a los usuarios a través de dichas herramientas.

En cuanto a la opinión de los participantes acerca de la utilidad y agrado de las llamadas de apoyo, los resultados obtenidos muestran que, a la gran mayoría de los participantes de la condición con apoyo, que sí habían recibido la llamada, les gustó y la consideraron útil. Sin embargo, aquellos de la condición sin apoyo, en la que no se realizó la llamada telefónica semanal, no la consideraron esencial. Detrás del hecho de que el grupo de participantes que no recibieron apoyo no valoraran tan positivamente el haber podido recibir una llamada, podría estar que consideraban que el programa ya les indicaba el ritmo que tenían que seguir, les animaba y les apoyaba, por lo que no la consideraron tan necesaria.

A la vista de estos resultados es importante tener en cuenta la necesidad de desarrollar no solamente intervenciones eficaces, sino que cuenten con aspectos que mejoren la satisfacción con la intervención (Wallin, Mattsson \& Olsson, 2016). En este sentido nos pueden ayudar estrategias que den apoyo a través de las TICs como aquellas con las que cuenta Sonreír es Divertido. La satisfacción y aceptación de los programas de intervención auto-aplicados es una variable fundamental a tener en cuenta en este tipo de investigaciones, ya que informa acerca de la viabilidad de las intervenciones y ayuda a optimizar la eficacia de las mismas (Wallin et al., 2016). Considerando las numerosas posibilidades de las TICs, es importante seguir estudiando estos aspectos. El apoyo automatizado a través de las TICs puede tener beneficios significativos e influir en los resultados, desempeñando una función similar al apoyo humano. La literatura muestra que los tratamientos auto-aplicados basados en Internet ahorran tiempo al terapeuta en comparación con los tratamientos tradicionales cara a cara. Por lo tanto, es posible que, al utilizar diferentes estrategias basadas en las TICs para proporcionar apoyo, el tiempo del terapeuta pueda reducirse aún más. Esto representa un progreso en la línea de la propuesta de Kazdin \& Blase (2011):la investigación en psicoterapia necesita desarrollar intervenciones que puedan aplicarse a más pacientes de una manera más simple y rentable.

Por tanto, respondiendo a la pregunta que se planteaba al inicio del estudio, se puede concluir que los resultados obtenidos en esta investigación sugieren la importancia de ofrecer apoyo durante la intervención auto-aplicada, aunque, también plantean que, en los programas con los que el usuario se siente satisfecho, el apoyo explícito se vuelve menos necesario.

\section{Referencias}

Bee, P. E., Bower, P., Lovell, K., Gilbody, S., Richards, D., Gask, L., \& Roach, P. (2008). Psychotherapy mediated by remote communication technologies: a meta-analytic review. BMC Psychiatry, 8, 60.

Beck, A. T., Steer, R.A., \& Brown, G.K. (1996). Manual for the Beck Depression Inventory-II. San Antonio, TX: Psychological Corp. 
Buntrock, C., Ebert, D. D., Lehr, D., Smit, F., Riper, H., Berking, M., \& Cuijpers, P. (2016). Effect of a web-based guided self-help intervention for prevention of major depression in adults with subthreshold depression: a randomized clinical trial. Jama, 315(17), 1854-1863.

Cuijpers, P., Donker, T., van Straten, A., Li, J., \& Andersson, G. (2010). Is guided self-help as effective as face-to-face psychotherapy for depression and anxiety disorders? A systematic review and meta-analysis of comparative outcome studies. Psychological Medicine, 40 (12), 1943-1957.

Donker, T., Blankers, M., Hedman, E., Ljotsson, B., Petrie, K., \& Christensen, H. (2015). Economic evaluations of Internet interventions for mental health: a systematic review. Psychological medicine, 45(16), 3357-3376.

Haro, J. M., Ayuso-Mateos, J. L., Bitter, I., Demotes-Mainard, J., Leboyer, M., Lewis, S. W., ... \& Robbins, T. W. (2013). ROAMER: roadmap for mental health research in Europe. International journal of methods in psychiatric research, 23(S1), 1-14.

Karyotaki, E., Riper, H., Twisk, J., Hoogendoorn, A., Kleiboer, A., Mira, A., ... \& Andersson, G. (2017). Efficacy of Self-guided Internet-Based Cognitive Behavioral Therapy in the Treatment of Depressive Symptoms: A Meta-analysis of Individual Participant Data. JAMA psychiatry, 74(4), 351-359.

Karyotaki, E., Kleiboer, A., Smit, F., Turner, D. T., Pastor, A. M., Andersson, G., ... \& Christensen, H. (2015). Predictors of treatment dropout in self-guided web-based interventions for depression: an 'individual patient data'meta-analysis. Psychological medicine, 45(13), 2717-2726.

Kazdin, A. E., \& Blase, S. L. (2011). Rebooting Psychotherapy Research and Practice to Reduce the Burden of Mental Illness. Perspectives on Psychological Science, 6 (1), 21-37.

Kazdin, A. E., \& Rabbitt, S. M. (2013). Novel Models for Delivering Mental Health Services and Reducing the Burdens of Mental Illness. Clinical Psychological Science, 1 (2), 170-191.

Kelders, S. M., Bohlmeijer, E. T., Pots, W. T., \& van Gemert-Pijnen, J. E. (2015). Comparing human and automated support for depression: Fractional factorial randomized controlled trial. Behaviour research and therapy, 72, 72-80.

Kessler, R. C. \& Greenberg. P. (2002). The economic burden of anxiety of anxiety and stress disorders, En L.D. Kennet, D. Charney, J. T. Coyle C. H. y Nemer- off (Eds.), Neuropsychopharmacology: the fifth generation of progress (pp. 981-991). Philadelphia: Lippincott Williams y Wilkins.

Melville, K. M., Casey, L. M., \& Kavanagh, D. J. (2010). Dropout from internet-based treatment for psychological disorders. British Journal of Clinical Psychology, 49, 455-471.

Mira, A. (2014). Eficacia diferencial de un programa de intervención auto-aplicado a través de Internet para la prevención y el tratamiento de la depresión leve o moderada con apoyo y sin apoyo por parte del terapeuta. Tesis Doctoral, Universitat Jaume I, Castellón, España.

Moritz, S., Schröder, J., Meyer, B., \& Hauschildt, M. (2013). The more it is needed, the less it is wanted: Attitudes toward face-to-face intervention among depressed patients undergoing online treatment. Depression and Anxiety, 30, 157-167.

Nathan, P. E., y Gorman, J. M. (Eds.). (2016). A Guide to Treatments That Work 4th Edition. New York: Oxford University Press.

Titov, N. (2011). Internet-delivered psychotherapy for depression in adults. Current opinion in psychiatry, 24(1), 18-23.

Wallin, E. E. K., Mattsson, S., \& Olsson, E. M. G. (2016). The Preference for internet-based psychological interventions by individuals without past or current use of mental health treatment delivered online: a survey study with mixed-methods analysis. JMIR mental health, 3(2), e25

World Health Organization (2017). Depression and Other Common Mental Disorders: Global Health Estimates. Geneva, Switzerland: WHO Document Production Services. 